\title{
Engrailed-1 Expression Marks a Primitive Class of Inhibitory Spinal Interneuron
}

\author{
Shin-ichi Higashijima, ${ }^{1,2}$ Mark A. Masino, ${ }^{1}$ Gail Mandel, ${ }^{1,2}$ and Joseph R. Fetcho ${ }^{1}$ \\ ${ }^{1}$ Department of Neurobiology and Behavior and ${ }^{2}$ Howard Hughes Medical Institute, State University of New York at Stony Brook, Stony Brook, New York \\ $11794-5230$
}

\begin{abstract}
Studies in chicks and mice have suggested that transcription factors mark functional subtypes of interneurons in the developing spinal cord. We used genetic, morphological, and physiological studies to test this proposed association in zebrafish. We found that Engrailed-1 expression uniquely marks a class of ascending interneurons, called circumferential ascending (CiA) interneurons, with ipsilateral axonal projections in both motor and sensory regions of spinal cord. These cells express the glycine transporter 2 gene and are the only known ipsilateral interneurons positive for this marker of inhibitory transmission. Patch recordings show that the CiA neurons are rhythmically active during swimming. Pairwise recordings from the CiA interneurons and postsynaptic cells reveal that the Engrailed-1 neurons produce monosynaptic, strychnine-sensitive inhibition of dorsal sensory interneurons and also inhibit more ventral neurons, including motoneurons and descending interneurons. We conclude that Engrailed-1 expression marks a class of inhibitory interneuron that seems to provide all of the ipsilateral glycinergic inhibition in the spinal cord of embryonic and larval fish. Individual Engrailed-1positive cells are multifunctional, playing roles in both sensory gating and motor pattern generation. This primitive cell type may have given rise to several, more specialized glycinergic inhibitory interneurons in birds and mammals. Our data support the view that the subdivision of spinal cord into different regions by transcription factors defines a primitive functional organization of spinal interneurons that formed a developmental and evolutionary foundation on which more complex systems were built.
\end{abstract}

Key words: interneuron; spinal cord; transcription factor; zebrafish; circuitry; inhibition

\section{Introduction}

Our understanding of the mechanisms of development of spinal interneurons has advanced rapidly in the last several years, with the identification of transcription factors associated with the regional differentiation of spinal cord. Studies of chicks and mice have shown that early in its development the spinal cord is divided from dorsal to ventral into discrete domains of neuronal precursors that express particular transcription factors (Briscoe et al., 2000; Jessell, 2000; Moran-Rivard et al., 2001; Pierani et al., 2001; Sharma and Peng, 2001). The precursors in these domains go on to produce postmitotic neurons identified by the expression of a different set of transcription factors. Evidence from transgenic mice indicates that the expression of some of these transcription factors, such as Engrailed-1 (En1), is restricted to a morphologically similar group of spinal interneurons (Burrill et al., 1997; Saueressig et al., 1999; Moran-Rivard et al., 2001).

This developmental work led to the important question of whether individual transcription factors might identify particular

\footnotetext{
Received Dec. 3, 2003; revised April 30, 2004; accepted April 30, 2004.

This work was supported by fellowships from Japan Science Corporation and the Japan Society for the Promotion of Science (S.H.), National Institutes of Health (NIH) Postdoctoral Fellowship NS44758 (M.A.M.), the Howard Hughes Medical Institute (G.M.), and NIH Grant NS26539 (J.R.F.). Drs. David McLean, Alan Roberts, and Steve Soffe provided helpful comments on this manuscript.

Correspondence should be addressed to Dr. Joseph Fetcho, Department of Neurobiology and Behavior, Life Science Building, State University of New York at Stony Brook, Stony Brook, NY 11794-5230. E-mail: Jfetcho@notes.cc.sunysb.edu.

DOI:10.1523/JNEUROSCI.5342-03.2004

Copyright $\odot 2004$ Society for Neuroscience $\quad 0270-6474 / 04 / 245827-13 \$ 15.00 / 0$
}

functional subtypes of spinal interneurons (Sharma and Peng, 2001). This is a fundamental question, not only because of its implications for how transcription factors may direct the differentiation of neuronal circuits, but also because the identification of genes, the expression of which is restricted to particular interneuron types, could lead to genetic tools for exploring the behavioral roles of these neurons. The promoters of cell type-specific genes would allow targeted expression of proteins that could be used to monitor or perturb the activity of particular classes of spinal interneurons (Fetcho, 2001; Sharma and Peng, 2001; Kiehn and Kullander, 2004). Such tools would set the stage for powerful studies of the links between neurons and behavior.

The only study directly addressing the question of the link between transcription factor and spinal interneuron type concluded that the population of spinal neurons marked by the transcription factor En1 in chicks is functionally heterogeneous (Wenner et al., 2000). This refutes the simple notion that transcription factors define interneuron types in all vertebrates. Not all vertebrates, however, have spinal cords as complex as those in birds and mammals. One possibility, raised by Goulding et al. (2002), is that transcription factors define a primitive functional organization of interneurons, one that might be revealed by studies of less complex vertebrates.

We used genetic, morphological, and physiological studies to explore the question of which interneurons are marked by the well studied transcription factor En1 (Eng1b in zebrafish) (Ekker et al., 1992; Force et al., 1999) in the simple spinal cord of embry- 
onic and larval zebrafish. Our data, together with the data of Li et al. (2004), reveal that En1 expression marks a single class of interneuron that appears to provide all of the ipsilateral glycinergic inhibition in embryos and larvae of fish and frogs. The interneurons share a similar overall dendritic and axonal morphology, with individual cells performing multiple functions in motor pattern generation and sensory gating. This "primitive" multifunction population may have diverged during the evolution of chicks and mammals to give rise to several different classes of ipsilateral glycinergic inhibitory interneurons with more restricted functional roles (Wenner et al., 2000). Our data support the view that the subdivision of spinal cord into different regions by transcription factors defines a primitive functional organization of spinal interneurons (a "ground state," as suggested by Goulding et al., 2002) that probably formed a developmental and evolutionary foundation on which more complex systems were built.

\section{Materials and Methods}

Cloning of the Eng1b genomic DNA. The reported Eng1b cDNA from zebrafish (Force et al., 1999) (GenBank accession number AF071237) lacked the $5^{\prime}$ region. To examine the putative transcriptional start site, $5^{\prime}$ RACE (rapid amplification of cDNA ends) was performed using two Englb-specific primers, which corresponded to the sequences near the 5' end of the reported region. We obtained a DNA fragment $\sim 300 \mathrm{bp}$ in length. This cDNA contained $\sim 250$ bp of the $5^{\prime}$ untranslated sequences and $50 \mathrm{bp}$ of the coding sequence. PCR analysis of the genomic DNA indicated that there is no intron in the corresponding region. The cDNA was used to screen a zebrafish genomic bacterial artificial chromosome (BAC) library (Incyte Genomics, Palo Alto, CA), and one BAC clone (see Fig. 2), $\sim 100 \mathrm{~kb}$ in length, was isolated. We have not generated a physical map of the BAC DNA, so the location of the Englb cDNA within the BAC is not reported.

Generation of the Eng-GFP expression vector. A PCR-amplified zebrafish $h s p 70$ promoter $(\sim 0.6 \mathrm{~kb})$ (Halloran et al., 2000$)$ was used as a basal promoter for the expression construct. Homologous recombination of BAC DNA was performed as described by Jessen et al. (1998). As a $5^{\prime}$ wing, $-3.1 \mathrm{~kb}$ of a genomic HindIII-XhoI fragment was used. The $\mathrm{XhoI}$ site is located $-0.8 \mathrm{~kb}$ upstream from the putative transcription start site (see Fig. 2 A). As a $3^{\prime}$ wing, $4.5 \mathrm{~kb}$ of a HindIII-HindIII fragment was used. The upstream HindIII site is located $0.9 \mathrm{~kb}$ downstream from the putative transcription start site (see Fig. $2 A$ ). The enhanced green fluorescent protein (EGFP) sequence was obtained from the pEGFP-N1 plasmid (Clontech, Palo Alto, CA). The SV40 poly(A) signal was extracted from the pcDNA1 plasmid (Invitrogen, Carlsbad, CA). The kanamycin-resistant gene was PCR amplified from the pAd10SacBII plasmid (Incyte Genomics). To make the final plasmid construct of the targeting vector for the homologous recombination, we subcloned, in this order, the $5^{\prime}$ wing, the $h s p 70$ promoter, EGFP, the SV40 polyA, the kanamycin-resistant gene, and the $3^{\prime}$ wing in pRM4-N (Jessen et al., 1998). The correct homologous recombination clones (see Fig. 2 A) were verified by restriction enzyme mapping.

A homologous recombination clone containing the endogenous Eng1b promoter was also generated. For this construct, the longer 5' wing, which contained the putative Englb promoter, was used in place of the $h s p 70$ promoter. The $3^{\prime}$ end of the wing corresponded to $150 \mathrm{bp}$ downstream from the putative transcription start site. There was no significant difference in GFP expression patterns with the two constructs (with or without the $h s p 70$ promoter). However, on average, the expression level of GFP appeared to be higher in the construct containing the hsp70 promoter. Therefore, the homologous recombination construct with the $h s p 70$ promoter was used for all of the experiments.

Microinjection of DNA. Microinjection of the DNA into zebrafish embryos was performed as described previously (Higashijima et al., 1997) using circular DNA at $40-50 \mathrm{ng} / \mu \mathrm{l}$.

For stochastic expression of cameleon (a derivative of GFP) (Miyawaki et al., 1997) in a limited number of neurons, the DNA constructs in which cameleon expression is driven by a goldfish neural tubulin promoter
(Hieber et al., 1998) or a zebrafish $H u C$ promoter (Park et al., 2000) were used as described previously (Higashijima et al., 2003). Approximately, one-third of the data was collected with the HuC promoter construct. The rest were collected with the goldfish neural tubulin promoter construct, which labeled fewer, more isolated cells.

Antibody and in situ hybridization staining. The anti-Engrailed antibody that was used in this study was a gift from A. Joyner (Skirball Institute, New York, NY). The antibody (anti-Enhb-1; rabbit polyclonal) was generated against the mouse En2 homeodomain and detects both En1 and En2 in mammals (Davis et al., 1991). In zebrafish, the antibody stains several groups of cells in the CNS, including a subset of neurons in the spinal cord (Hatta et al., 1991). Because Eng1b is the only engrailedrelated gene expressed in spinal neurons, the protein recognized by the antibody in spinal neurons ought to be Englb. Consistent with this, staining patterns of En1 antibody (anti-Enhb-1) and Eng1b in situ hybridization in the developing spinal cord were identical.

Standard whole-mount antibody staining procedures were used with Cy5-conjugated anti-rabbit secondary antibody (Jackson ImmunoResearch, West Grove, PA). For animals older than $36 \mathrm{hr}$, the ventral region (below the notochord) was cut off for better penetration of the antibodies. For dual staining with the antibody and in situ hybridization, samples were first treated with the antibodies. All of the solutions contained RNase inhibitor (Roche, Indianapolis, IN). After postfixation, wholemount in situ hybridization was performed with standard procedures, except for the omission of the proteinase treatment. The staining was detected with alkaline phosphatase by using a fluorescent substrate [2-hydroxy-3-naphtoic acid-2' -phenylanilide phosphate (HNPP) FastRed; Roche], which yielded rhodamine-like fluorescence. Probes for in situ hybridization were a mixture of vesicular glutamate transporters (VGLUT2.1 and VGLUT2.2), glycine transporter2 (GLYT2), and a mixture of GAD65 and GAD67 (Martin et al., 1998). The VGLUT genes and GLYT2 were isolated by us. They have high sequence homology to their mammalian counterparts. The details of their cloning and expression patterns will be described elsewhere. The length of the probes used for VGLUT2.1 was $1.4 \mathrm{~kb}$ with $0.1 \mathrm{~kb}$ of the $5^{\prime}$ untranslated region (UTR) and $1.3 \mathrm{~kb}$ of the coding sequence (74\% of the coding region). VGLUT2.2 was $1.6 \mathrm{~kb}$ with $0.3 \mathrm{~kb}$ of the $5^{\prime}$ UTR and $1.3 \mathrm{~kb}$ of the coding sequence (74\% of the coding region). GLYT2 was $1.7 \mathrm{~kb}$ with $0.3 \mathrm{~kb}$ of the $5^{\prime}$ UTR and $1.4 \mathrm{~kb}$ of the coding sequence (62\% of the coding region). GAD67 was $1.5 \mathrm{~kb}$ with $0.3 \mathrm{~kb}$ of the $5^{\prime}$ UTR and $1.2 \mathrm{~kb}$ of the coding sequence ( $65 \%$ of the coding sequence). For GAD65, it was $0.7 \mathrm{~kb}$, the same sequence shown by Martin et al. (1998).

Standard cryostat sectioning was performed to obtain cross sections $(20 \mu \mathrm{m})$ of spinal cord. Briefly, samples were soaked in 30\% sucrose, mounted in Tissue-Tek optimal cutting temperature compound (Sakura Finetek, Torrance, CA), and were frozen before sectioning. During this procedure, the tissue shrank to $\sim 70 \%$ of its original size. To compare the sectioned images with unsectioned ones, sectioned images were enlarged to match the unsectioned ones.

Retrograde labeling. Retrograde labeling of spinal motoneurons or interneurons was performed as described previously (Fetcho and O'Malley, 1995; Hale et al., 2001). Rhodamine-conjugated dextran (M.W. 3000; Molecular Probes, Eugene, OR) was used as a dye. For labeling motoneurons, the dye was pressure injected into ventral muscle. For interneurons, the dye was pressure injected into the spinal cord.

Microscopic observation of zebrafish neurons. All of the microscopic observations in this study were carried on a confocal microscope (LSM510; Zeiss, Thornwood, NY). For living fish, a Plan-Neofluor $25 \times$ multi-immersion lens [numerical aperature (NA), 0.80; Zeiss] was used with water immersion and a coverslip. For the observation of fixed tissues (antibody staining or in situ hybridization), a $63 \times \mathrm{C}$-Apochromat lens (NA, 1.20; Zeiss) was used. All of the images were taken with the fish embedded on their sides in low melting point agarose (1.2\%). An argon laser of $488 \mathrm{~nm}$ was used for the observation of GFP or cameleon, a helium-neon laser of $543 \mathrm{~nm}$ for observation of rhodamine and HNPP/ Fast-red, and a helium-neon laser of $633 \mathrm{~nm}$ for observation of Cy5.

Electrophysiological methods. Zebrafish larvae (4-6 d old) with neurons expressing GFP under the control of the En 1 promoter were anesthetized with $0.2 \%$ Tricaine-S (Western Chemical International, Scotts- 
dale, $\mathrm{CA}$ ) in an extracellular recording solution that contained (in $\mathrm{mm}$ ) $134 \mathrm{NaCl}, 2.9 \mathrm{KCl}, 1.2 \mathrm{MgCl}_{2}, 2.1 \mathrm{CaCl}_{2}, 10 \mathrm{HEPES}$ buffer, and 10 glucose, adjusted to pH 7.8 with $\mathrm{NaOH}$ (Legendre and Korn, 1994; Drapeau et al., 1999). The preparations were paralyzed with $0.01 \mathrm{~mm}$ D-tubocurarine (Sigma, St. Louis, MO) added to the recording solution, which was bubbled with ambient air and superfused continuously at $\sim 22-26^{\circ} \mathrm{C}$

Larvae were pinned to a Sylgard-lined glass-bottomed Petri dish with short pieces $(\sim 2 \mathrm{~mm})$ of fine tungsten wire (0.001 inches) pushed through the notochord, one pin placed near the air bladder and another near the anus. The skin between the two pins was removed with a pair of fine forceps. Collagenase $(0.1 \%$; Sigma) in recording solution was applied to the preparation for $3-5$ min to enzymatically prepare the muscle fibers for easier removal. The collagenase solution was washed off, and a large bore $(\sim 15 \mu \mathrm{m}$ in diameter) glass microelectrode attached to an extracellular suction electrode holder was used to aspirate away individual muscle fibers overlying a small section (two to three segments) of the spinal cord. For all electrophysiology experiments, the preparations were observed using a water immersion objective ( $40 \times$; NA, 0.80; Olympus, Melville, NY) on an upright microscope (BX51WI; Olympus) fitted with differential interference contrast optics.

Extracellular recording techniques were used to monitor the activity of peripheral nerves during fictive swimming at $\sim 22-26^{\circ} \mathrm{C}$. Extracellular suction electrodes $(\sim 10-15 \mu \mathrm{m}$ in diameter $)$ pulled on a FlamingBrown micropipette puller (P-97; Sutter Instruments, Novato, CA) from borosilicate glass [ $1.5 \mathrm{~mm}$ outer diameter $(\mathrm{OD}) ; 1.12 \mathrm{~mm}$ inner diameter (ID); A-M Systems Inc., Carlsborg, WA) were filled with curare-free extracellular recording solution (see above) and placed in a suction electrode holder [E series (Warner Instruments, Hamden, CT) or HL-U (Axon Instruments, Union City, CA)]. The tip of the suction electrode was positioned at the dorsoventral midpoint of a myotomal cleft where the skin had been removed, and a light suction was applied to ensure a tight seal with the underlying muscle tissue and peripheral nerves. In early experiments, extracellular signals were monitored with a differential AC amplifier (model 1700; A-M Systems) at a gain of 10,000 with the low- and high-frequency cutoff set at 300 and $500 \mathrm{~Hz}$, respectively. Noise was reduced with a $60 \mathrm{~Hz}$ notch filter. In most experiments, however, a MultiClamp 700A (Axon Instruments) amplifier was used to monitor extracellular signals in voltage-clamp mode at a gain of 1000 with the low- and high-frequency cutoff at 100 and $4000 \mathrm{~Hz}$, respectively.

Standard whole-cell recording techniques were used to monitor the activity of interneurons in vivo at $\sim 22-26^{\circ} \mathrm{C}$. Patch electrodes $(\sim 15 \mathrm{M} \Omega)$ were pulled on a Flaming-Brown micropipette puller (P-97; Sutter Instruments) from borosilicate glass ( $1.5 \mathrm{~mm}$ OD; $0.86 \mathrm{~mm}$ ID; Warner Instruments) and were filled with patch solution (in mM): $125 \mathrm{~K}$ gluconate, $2 \mathrm{MgCl}_{2}, 10 \mathrm{HEPES}$ buffer, $10 \mathrm{EGTA}$, and $4 \mathrm{Mg}$ ATP, adjusted to pH 7.2 with $\mathrm{KOH}$. The calculated junction potential (using Clampex; Axon Instruments) for our solutions is $16 \mathrm{mV}$. We did not correct for the junction potential in the data we present. The correction would shift our measured potentials $16 \mathrm{mV}$ more negative and would not alter our conclusions. In some previous experiments, we used a different patch solution (in $\mathrm{mM}$ ): $115 \mathrm{~K}$ gluconate, $15 \mathrm{KCl}, 2 \mathrm{MgCl}_{2}, 10$ HEPES buffer, 10 EGTA, and $4 \mathrm{Mg}$ ATP, adjusted to pH 7.2 with $\mathrm{KOH}$, with a calculated junction potential of $15 \mathrm{mV}$. Positive pressure $(30-50 \mathrm{mmHg})$ was applied to the patch electrode as it approached the exposed surface of the spinal cord. The tip of the electrode was lowered carefully until it broke into the cord. Interneurons were targeted for recording based either on their size, shape, and position in the spinal cord [commissural primary ascending interneurons (CoPAs)] or on their expression of GFP (En1positive CiAs). Release of positive pressure formed a gigaohm seal once the tip of the patch electrode was directly apposed to the appropriate interneuron. Suction pulses were applied to break the seal for whole-cell voltage recordings. Whole-cell voltage was monitored with a MultiClamp 700A (Axon Instruments) amplifier at a gain of $20\left(\mathrm{R}_{\mathrm{f}}=5 \mathrm{G} \Omega\right.$ ), filtered at $30 \mathrm{kHz}$ and digitized at $63 \mathrm{kHz}$. The recordings were accepted for data analysis if the resting membrane potential was more negative than $-45 \mathrm{mV}$ (range, -46 to $-70 \mathrm{mV}$; mean, $-58.5 \pm 6.0 \mathrm{mV} ; n=33$ total cells: 18 CiAs, 15 CoPAs). Neurons were labeled with $0.1 \%$ Sulforhodamine B (Sigma) in the patch solution, and fluorescent images were acquired with a CCD camera (C-72-CCD; Dage MTI, Michigan City, IN) and a frame grabber (LG3; Scion, Frederick, MD) controlled by NIH Image software for morphological identification of the neurons. Wholecell voltage recordings were used to determine the synaptic reversal potential (measured at the soma) in CoPA interneurons held, by continuous current injection, at various depolarized membrane potentials during fictive swimming initiated by light stimulation. In some experiments, strychnine (1-5 $\mu \mathrm{M}$; Sigma) was applied briefly (1-2 min) to the extracellular solution bathing the preparation to block glycinergic synapses.

The connections between CiA interneurons and more ventral motoneurons and interneurons were more difficult to find than connections with dorsal CoPA interneurons (see Results). To allow for testing of more potential postsynaptic cells, in some cases, we used a loose patch onto the GFP-filled CiA interneuron without going whole cell. This allowed us to stimulate it to fire with a voltage step while doing simultaneous wholecell recordings from candidate postsynaptic neurons with cell bodies located near the axonal branches of the CiA neuron. In these cases, we were confident that we were stimulating the CiA neuron because we could see GFP from the portion of it sucked into the patch pipette, and we could also record the currents from action potentials in the CiA neuron in response to our stimulus.

Extracellular and whole-cell recordings were digitized (DigiData series 1322A; Axon Instruments), acquired using pClamp 8.2 software (Axon Instruments), and analyzed offline with Clampfit 8.2 (Axon Instru-
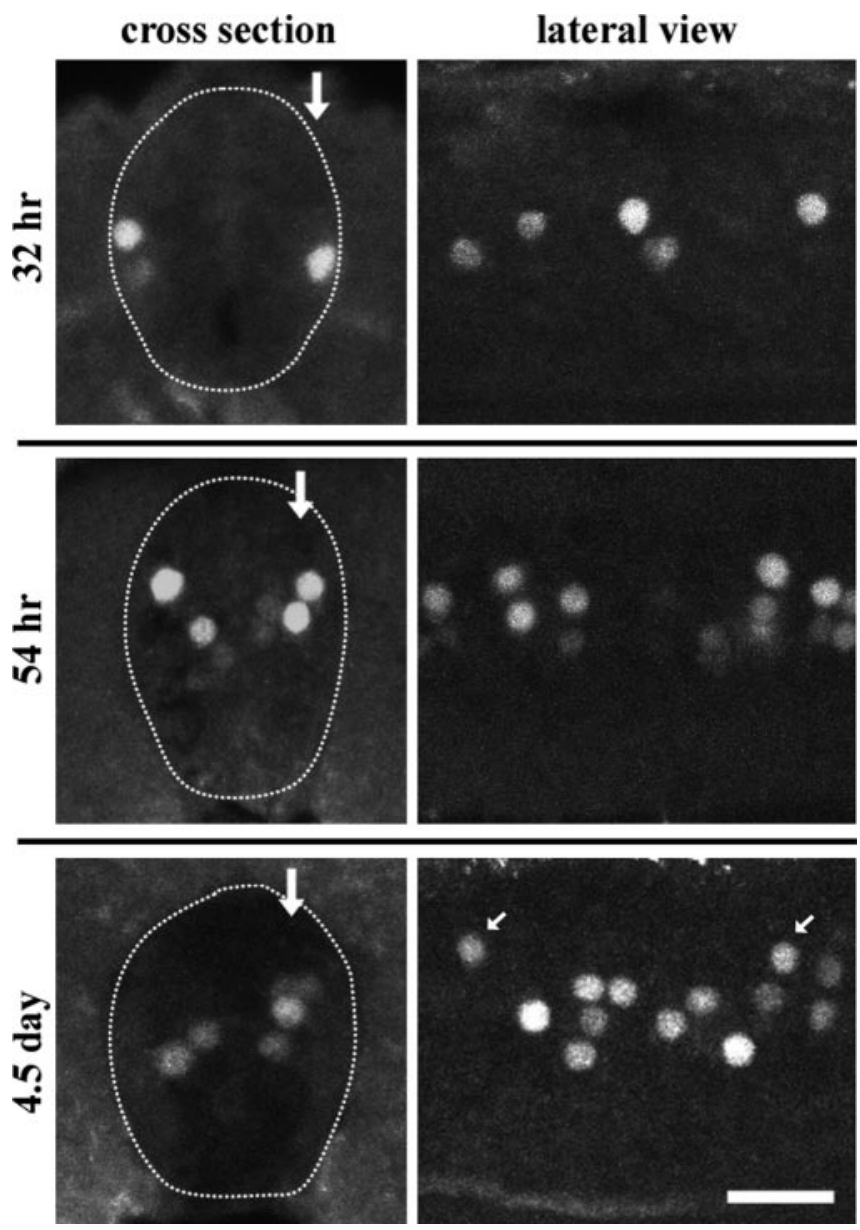

Figure 1. En1 expression in embryos and larvae. Spinal cords at three different times $(32 \mathrm{hr}$, $54 \mathrm{hr}$, and $4.5 \mathrm{~d}$ ) were stained with antibody to En proteins (called anti-En 1 antibody hereafter). The left panels show cross sections of spinal cords (dotted lines outline the boundary of the cord), whereas the right panels show a lateral view of confocal optical sections. Arrows in the left panels show the level of optical sections in the corresponding right panels. Dorsal is at the top. In the right panels, rostral is to the left. Scale bar, $20 \mu \mathrm{m}$. 
A Enl BAC

(approximately, $100 \mathrm{~kb}$ )

putative basal promoter

$1 \mathrm{~kb}$

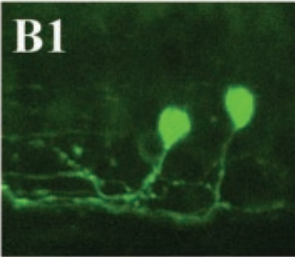

Targeting construct

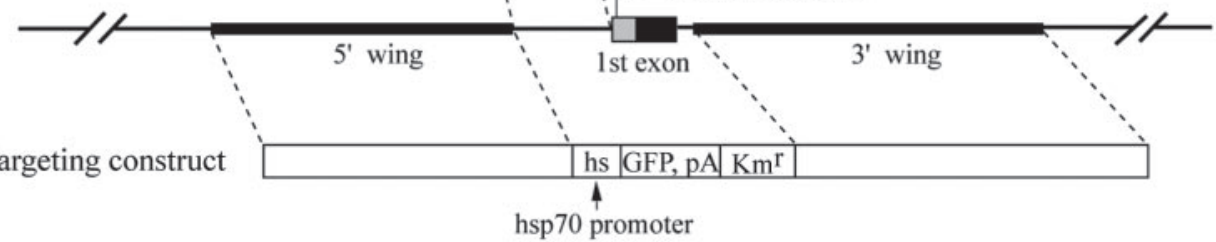

$\longrightarrow$

$\rightarrow$ Enl transcription

En1-GFP BAC

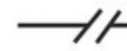

5' wing

\begin{tabular}{|l|l|l|}
\hline hs & $\mathrm{GFP}, \mathrm{pA}$ & $\mathrm{Km}^{\mathrm{r}}$ \\
\hline
\end{tabular}

3' wing
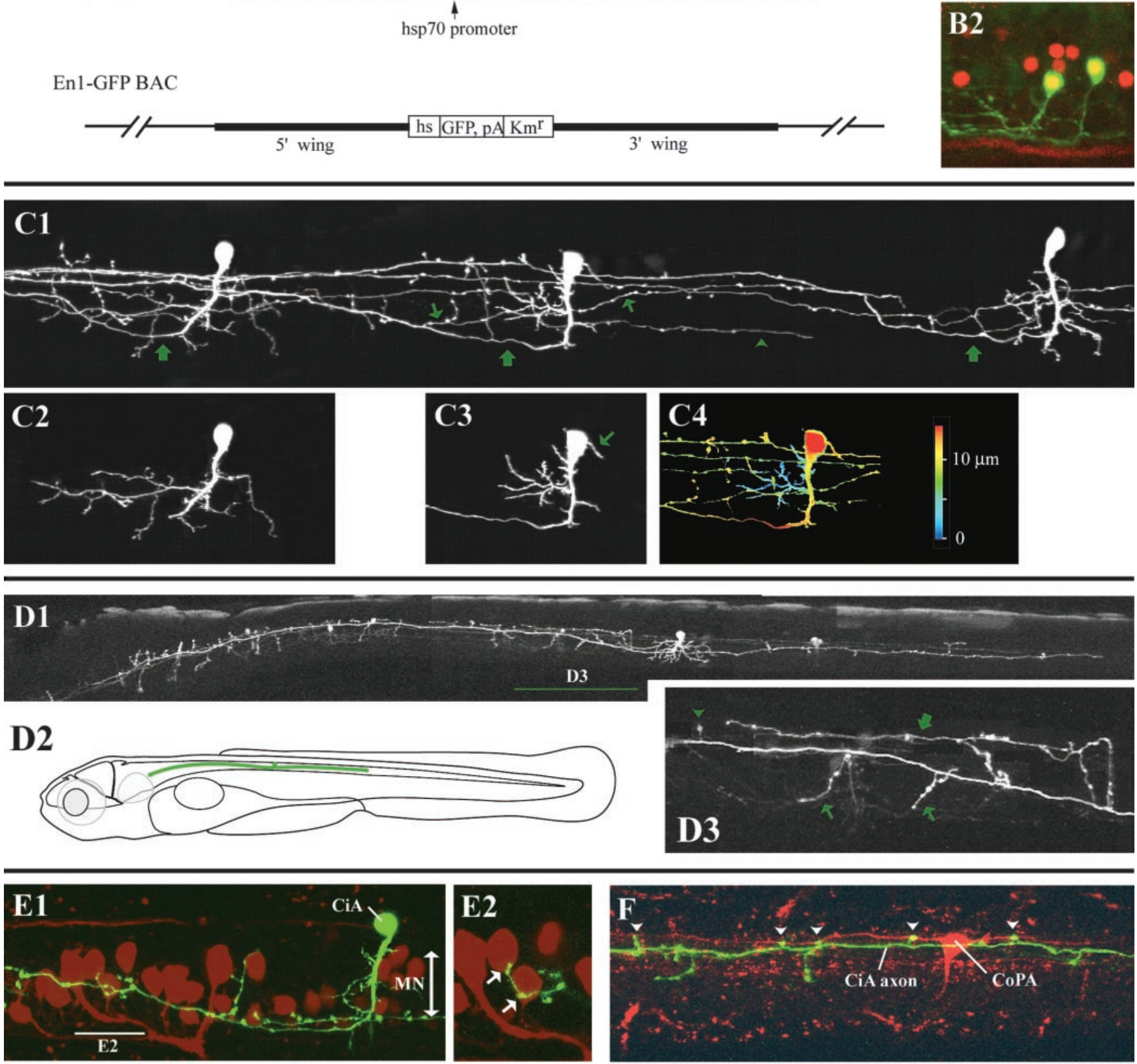

Figure 2. BAC GFP expression construct and morphology of the En1-positive interneurons. A, Construction of the En1-GFPBAC (BAC). The top drawing shows the structure of the BAC, which covers the En1 (eng1b) locus. The box shows the first exon of En1, with the open box being the 5' UTR and the filled box the coding region. The middle drawing shows a schematic of the targeting vector for homologous recombination in bacteria ( $\mathrm{Km}^{\mathrm{r}}$, kanamycin resistance gene). The bottom drawing shows the structure of the En1-GFP BAC used for microinjection into zebrafish embryos. $B-F$ show the morphology of En1 interneurons from fish at $4-5 \mathrm{~d}$ of age. Dorsal is to the top, and rostral is to the left, as in all of the figures. All of the images, except for $B 1, B 2$, and $D 2$, were taken from live animals. B1, B2, GFP-labeled CiA interneurons in the DNA-injected fish. In B2, the sample was counterstained with the anti-En 1 antibody, showing that the GFP-labeled CiA interneurons (green) were positive for En1 (red). C1, Three GFP-labeled CiA interneurons are shown. Confocal optical sections are stacked to show the entire morphology of the cells. Thick arrows indicate primary ascending axons from the three cells. Small arrows show a descending axon from the middle cell, which arises from the ascending portion of the main axon (near the left small arrow). This cell also has an additional descending axon (arrowhead). C2, C3, Confocal optical sections were combined to show the dendritic morphology of the left (C2) and the center cell (C3) shown in C1. Dendrites arise from the ventrally extending main process. CiA interneurons are typically unipolar, but occasionally small dendrites arise directly from the soma (C3, arrow). C4, Depth coding of the confocal optical sections of the center cell in (1. Dendrites are mostly superficial (blue color) in the marginal zone. D1, The entire morphology of a CiA interneuron. All of the clearly labeled processes are from the CiA interneuron, although a small number of other very weakly labeled cells are present. The rostrally extending axon of the CiA is more thickly labeled than the caudally extending one. D2, A schematic drawing to show the region where the axons of the CiA interneuron in D1 run in the fish. Both ascending and descending axons extend for multiple segments, with the (Figure legend continues.) 
ments) and/or Matlab 5.3 (Mathworks, Natick, MA). An isolated pulse stimulator (model 2100; A-M Systems) was used to deliver current pulses into targeted interneurons. The amplitude and duration of the current pulse were adjusted for each cell to fire a single-action potential per stimulus. Current pulses were applied at low frequencies $(0.1-1.0 \mathrm{~Hz})$ to determine the firing threshold of the presynaptic neurons and at increasingly higher frequencies $(1.0-5.0 \mathrm{~Hz})$ to explore how well the postsynaptic potentials (PSPs) followed presynaptic firing and to determine whether there was jitter in latency that would suggest a polysynaptic connection.

\section{Results}

\section{Engrailed genes in zebrafish}

Mammals have two Engrailed-related genes, En1 and En2 (Joyner and Martin, 1987). En1 is expressed in a subset of developing spinal interneurons, and thus the homologs of $E n 1$ were the focus of our study. Zebrafish have two En1-related genes (Eng1 and Eng1b) (Ekker et al., 1992; Force et al., 1999) along with two En2-related genes (Eng2 and Eng3) (Holland and Williams, 1990; Fjose et al., 1992). Of the two Eng1 genes, only Eng1b is expressed in the spinal cord (Ekker et al., 1992; Force et al., 1999). We therefore focused on Englb in this study. For simplicity, by analogy to mammals, we use the term En1 in place of Englb throughout the text.

\section{Distribution of En1-positive cells in the spinal cord between $32 \mathrm{hr}$ and $5 \mathrm{~d}$}

Previous studies have only described En 1 expression in the spinal cord in early zebrafish embryos (Hatta et al., 1991; Force et al., 1999). We therefore first examined the developmental profile of En1 expression from early embryo until after the fish become free swimming at $4-5 \mathrm{~d}$ to determine which cells were labeled and how their morphology changed during development. Because there is a rostrocaudal developmental gradient in the spinal cord, especially at early stages, we focused on one region at midbody, from approximately segments 6-13.

Weak En1 expression in a small number of cells was first detected at $\sim 20 \mathrm{hr}$ (data not shown) (Hatta et al., 1991). During development, both the intensity of En 1 staining and the number of En1-expressing cells increased. Figure 1 (top panels) shows the expression pattern in $32 \mathrm{hr}$ embryos. Postmitotic neurons are located only in the lateral region of the spinal cord at this stage. Consistent with this, all of the En1-positive cells are also located near the lateral margin. As development proceeds, more and more En1-positive cells are added. Figure 1 (middle panels) shows the expression pattern in $54 \mathrm{hr}$ embryos, when intensely labeled cells tend to be located laterally, whereas lightly labeled cells are more medial. The lightly labeled cells in the medial region are most likely newly added, young En 1-positive cells. Many lightly labeled cells were found in the medial region at this stage, suggesting that differentiation of En1-positive cells is highest around this time. Figure 1 (bottom panels) shows the expression pattern in 4.5-d-old larvae. At this stage, there is no differential labeling along the lateral-medial axis, suggesting that proliferation of En1-positive cells is mostly over. Throughout all of the stages, most of the En1-positive cells were located at intermediate levels along the dorsoventral extent of cord, although occasional
En1-positive cells were found more dorsal in the spinal cord (Fig. 1 , bottom right, arrows). The staining intensity in some cells was extremely weak at 4-5d, suggesting that En 1 expression in some cells is being downregulated by $5 \mathrm{~d}$.

\section{Visualization of En1-positive cells in living fish}

To visualize En1-positive cells in live animals for morphological and physiological studies, we expressed GFP under the control of cis-regulatory sequences from the En1 gene (Fig, 2A, top). The GFP-modified BAC DNA (Fig. 2A, bottom, En1-GFP BAC) was microinjected into one-cell stage zebrafish embryos (Higashijima et al., 1997), and GFP expression was examined at 3-5 d. With this approach, the transgene is stochastically retained by a small number of cells at later stages. Consequently, a small, random subset of the En1-positive cells was expected to express GFP.

Among the DNA-injected fish, many had GFP labeling in neurons with a primary axon that first extends ventrally, turns rostrally in ventral spinal cord, and then ascends on the ipsilateral side of the spinal cord among other axons in the marginal zone lateral to the motoneurons, moving more dorsally as it ascends (Fig. 2B1,C1). The morphology of an interneuron like this, present early in zebrafish development, was briefly described previously (Bernhardt et al., 1990); we use the name circumferential ipsilateral ascending (CiA) interneuron, assigned in that study.

Approximately $65 \%$ of the neurons labeled by the BAC injections were $\mathrm{CiA}$ interneurons. The rest were mostly commissural interneurons, but other types of neuron, such as ipsilateral descending interneurons or Rohon-Beard (RB) sensory neurons, were sometimes labeled. Among these non-CiA neurons, RB sensory neurons were expected to be negative for En1 based on the location of the neurons, which is well dorsal to the neurons labeled by En1 antibody staining. Thus, the construct appeared to be somewhat leaky, and this led us to suspect that the GFP labeling in the less abundant, non-CiA-type neurons might be spurious.

To address this issue, we counterstained the GFP-labeled interneurons with the anti-En1 antibody. In 3-d-old fish, all of the GFP-labeled CiA interneurons were positive for En1 $(n=53)$. In contrast, all of the GFP-labeled neurons belonging to other types were negative $(n=22)$, indicating that labeling in non-CiA neurons was indeed attributable to leaky expression. Similar results were obtained in 4- to 5-d-old fish. At this age, the vast majority of CiA interneurons (22 of 24) were positive for En1. An example of this experiment is shown in Figure 2, B1 and B2. Two interneurons of the 24 we examined at $4-5 \mathrm{~d}$ did not have a detectable level of En1 staining. We speculate that En1 expression in these interneurons had already been downregulated below detection by this stage. At 4-5 d, the GFP-labeled, non-CiA-type neurons were always negative for En1 $(n=10)$, as in the 3 -d-old fish.

These observations showed that En1 is expressed in CiA interneurons. Although leaky, the BAC homologous recombination construct was able to drive GFP expression in many En1-positive $\mathrm{CiA}$ interneurons in living fish, which allowed for more detailed in vivo structural and functional studies.

(Figure legend continued.) rostral axon ending in the hindbrain. D3, Close-up view of the region marked in D1 by the bar labeled D3. Several processes are evident. These include a long dorsally extending process (thickarrow), a small dorsally extending one (arrowhead), and two ventrally extending ones (arrows). E1, One GFP-labeled CiA (green) and retrogradely labeled motoneurons (ventral red cells) are shown. Ventral processes from the CiA interneuron extend among the motoneurons and appear to contact their somata, as shown in E2 (arrows) in a higher magnification view of a narrow optical section medial to the main axon. F, A caudally extending axon from a GFP-labeled CiA interneuron (green) and a retrogradely labeled CoPA interneuron (red) are shown. Dorsally extending processes from the axon have apparent contacts onto the CoPA dendrites (arrowheads). Ventrally extending processes also arise from the axon. Scale bars: $B 1, B 2, C 1-C 4, D 3, E 1, F, 50 \mu \mathrm{m} ; E 2,25 \mu \mathrm{m} ; D 1,160 \mu \mathrm{m} ; D 2,670 \mu \mathrm{m}$. 


\section{Morphology of En1-positive \\ CiA interneurons}

We examined the morphology of 55 GFPlabeled $\mathrm{CiA}$ interneurons in confocal optical sections from 4- to 5-d-old (Fig. 2C-F) living fish. The cells were examined in midbody regions from segments $7-14$. The somata of CiA interneurons were located in the intermediate to dorsal region of the spinal cord. Along the lateral-medial axis, some were located more superficially, with others medial to them. The distribution of GFP-labeled CiA interneurons matched well with the distribution of En1-positive cells revealed by the antibody staining (Fig. 1 , bottom).

The CiA interneurons were predominantly unipolar, with occasional additional small processes on the soma (Fig. $2 C 3$, arrow). The thick, primary process came off from the ventral pole of the soma and extended ventrally. The process gave rise to extensive dendrites lying mainly in the middle and ventral parts of the marginal zone. Figure $2 C 4$ is a depth coded stacked image that shows that the majority of dendrites were superficial to the main process. The most ventral dendrites sometimes turned medially and extended near the floor of the spinal cord. Some neurons had larger dendritic fields (Fig. 2C2) than others (Fig. 2C3), but there was a range of sizes rather than discrete categories. The ventrally extending primary process from the soma became an axon that turned rostrally in ventral spinal cord and extended rostrally and dorsally just lateral to the motor column (Fig. 2C1, thick arrows). The typical CiA interneuron had one ascending axon, with a descending axonal branch that arose from the proximal portion of the ascending axon (Figs. $2 C 1$, short arrows, 3 ). In some cases, the descending branch arose near the most ventral part of the main axon where the primary axon turned to ascend. All 55 of the CiA interneurons examined had at least one descending axonal branch; occasionally, there was more than one (Figs. 2C1, arrows and arrowhead, 3).

In seven fish, well isolated, single $\mathrm{CiA}$ interneurons were labeled, allowing us to clearly follow their entire axonal trajectory. In these fish, both the ascending and descending portions of the axon typically ran over multiple segments. An example is shown in Figure 2D1. The ascending portions of the axon were always more densely labeled than the descending ones, suggesting that the ascending axons were of larger caliber. In most cases (six of seven), the ascending branches were longer than descending ones. The average length of the axons was $\sim 1050 \mu \mathrm{m}$ for the ascending part $(\sim 10$ segments in length) and $750 \mu \mathrm{m}$ for the descending ( $\sim 7$ segments in length). The ascending axons of these neurons extended near or into hindbrain, like the CiA interneuron shown in Figure 2D1, the axon of which ended just caudal to the level of the ear. Except for the region near the soma, both the ascending and descending portions of the axon ran dorsal to the motor column and slightly ventral to the sensory tracts formed by the axons of RB sensory neurons and dorsal root ganglion neurons.

Along their trajectory, both the ascending and the descending axonal branches budded off processes or collaterals both ventrally and dorsally into presumptive motor and sensory regions (Fig. $2 D 1, D 3, E, F$ ). These branches, as well as the primary axon, had swellings that are probable sites of synaptic contact. The anatomical relationships between the CiA axons and both motoneurons and sensory interneurons were more carefully examined in double-labeling experiments. When motoneurons were retrogradely labeled by injection of rhodamine dextran into the muscle of fish with GFP-filled CiA neurons, we found processes arising from the main axon of the CiA interneurons that extended medially into the motor column and appeared to contact the somata of motoneurons (Fig. 2E). More laterally directed branches from the CiA axon were located where they could contact dendrites of motoneurons, which extend laterally from their somata. We used a similar double-labeling approach to examine the morphological relationships between the $\mathrm{CiA}$ interneurons and one class of sensory interneuron, the CoPA. The CoPA interneurons receive inputs from primary sensory afferents (Gleason et al., 2003) and relay excitation to the opposite side of the spinal cord. The dorsal portions of the CiA axon appeared to contact the somata and the longitudinal dendrites of the CoPA interneurons (Fig. 2F). The branching pattern of isolated CiA interneurons suggested that individual cells contacted both motoneurons via their ventral branches and the CoPA interneurons via their more dorsal processes. Their branches were in locations where they could also contact other known classes of interneurons, such as the descending interneurons and commissural inhibitory interneurons.

The CiA interneurons examined in this study were morphologically homogeneous, with no discrete categories evident based on broad visual inspection. They all shared the following basic features: (1) intermediate to dorsal soma position; (2) ventrally extending initial process from which dendrites came off laterally; (3) rostrally extending primary axon that extended dorsally; and (4) a caudally extending axonal branch. There was some variation in the extent of dendritic branching and the axonal trajectory, with the rostrally extending axons from some CiA interneurons running dorsally more quickly than others. There was, however, a continuum in morphology with no clearly distinguishable subtypes.

\section{Development of $\mathrm{CiA}$ interneurons}

The trajectories of the CiA interneurons in 4- to 5-d-old fish suggested that the ascending axons were the primary ones. We 
therefore suspected that the ascending axons might have extended first, with the descending branch added later during development. To address this issue, we performed time-lapse analysis to follow the development of GFP-labeled CiA interneurons. As expected, CiA interneurons at early stages sometimes had only ascending axons. In the cases in which they had descending branches, the descending branches tended to be very short. Figure 3 shows an example of such a time-lapse analysis. At the first time point $(40 \mathrm{hr})$, the ascending axon from the right cell already extended for multiple segments (the rostral end of the ascending axon is out of the picture), whereas two descending branches from this cell were very short (Fig. 3, top, thick and thin arrows). In particular, the descending branch arising from the ascending axon was extremely tiny (thin arrow), suggesting that this branch had just formed. During subsequent development, these two descending axonal branches grew and ultimately ran for several segments (Fig. 3, bottom, arrows). A similar developmental pattern was observed in the more rostral interneuron (Fig. 3, left cell). Thus, the ascending axons appear to form first, followed quickly by the development of descending branches.

\section{All interneurons having a CiA morphology are En 1 positive}

Although the BAC construct and En1 staining revealed that En1 is expressed in at least some CiA interneurons, there were two important questions that remained concerning the definition of the En1-positive cells in the spinal cord. The first was whether En1 is expressed in all interneurons having CiA-like morphology. Although En1 was expressed in the CiA interneurons revealed by the En1-GFP BAC construct, we could not, based on the previous experiment, rule out the possibility that there might be CiA-like interneurons in which En 1 is not expressed. The second question was whether En 1 is expressed only in CiA interneurons. The previous experiments did not allow us to rule out the possibility that the sequences in the En1-GFP BAC lacked regulatory elements responsible for directing En1 expression in a different type of interneuron that would then have been missed by the BAC construct.

To address these questions, we labeled a variety of types of neurons in a more unbiased way and examined whether neurons having a particular morphology were positive or negative for En 1 . For this purpose, pan-neuronal promoters (goldfish neural tubulin promoter and zebrafish $\mathrm{HuC}$ promoter) were used to drive transient expression of cameleon (a genetically encoded calcium indicator derived from GFP used here just to examine morphology) (Miyawaki et al., 1997; Higashijima et al., 2003) to stochastically label many different classes of spinal neurons. We used 32-36 hr animals instead of 4- to 5-d-old fish in this experiment because the stochastic expression led to many labeled cells that were easily identified at early stages but that were more difficult to distinguish after more extensive dendritic and axonal arbors had developed by $4-5 \mathrm{~d}$ (Fig. 3, compare $40 \mathrm{hr}$ and $68 \mathrm{hr}$ ).

We found three classes of ipsilateral ascending interneurons labeled by transient expression with pan-neuronal promoters, which is consistent with results from our other studies of the cell types in the spinal cord (our unpublished observations). These are $\mathrm{CiA}$ interneurons, dorsal longitudinal ascending interneurons (DoLA), and Kolmer-Agduhr (KA) interneurons [these were originally described by Bernhardt et al. (1990) and Kuwada et al. (1990)]. The detailed morphology of DoLA and KA interneurons will be described elsewhere. Briefly, DoLA interneurons were defined by their very dorsal soma (near the top of the spinal cord) and straight, ascending axons located at the level of sensory tracts (Fig. 4C1,D1, dorsal cell). The KA interneurons were de-
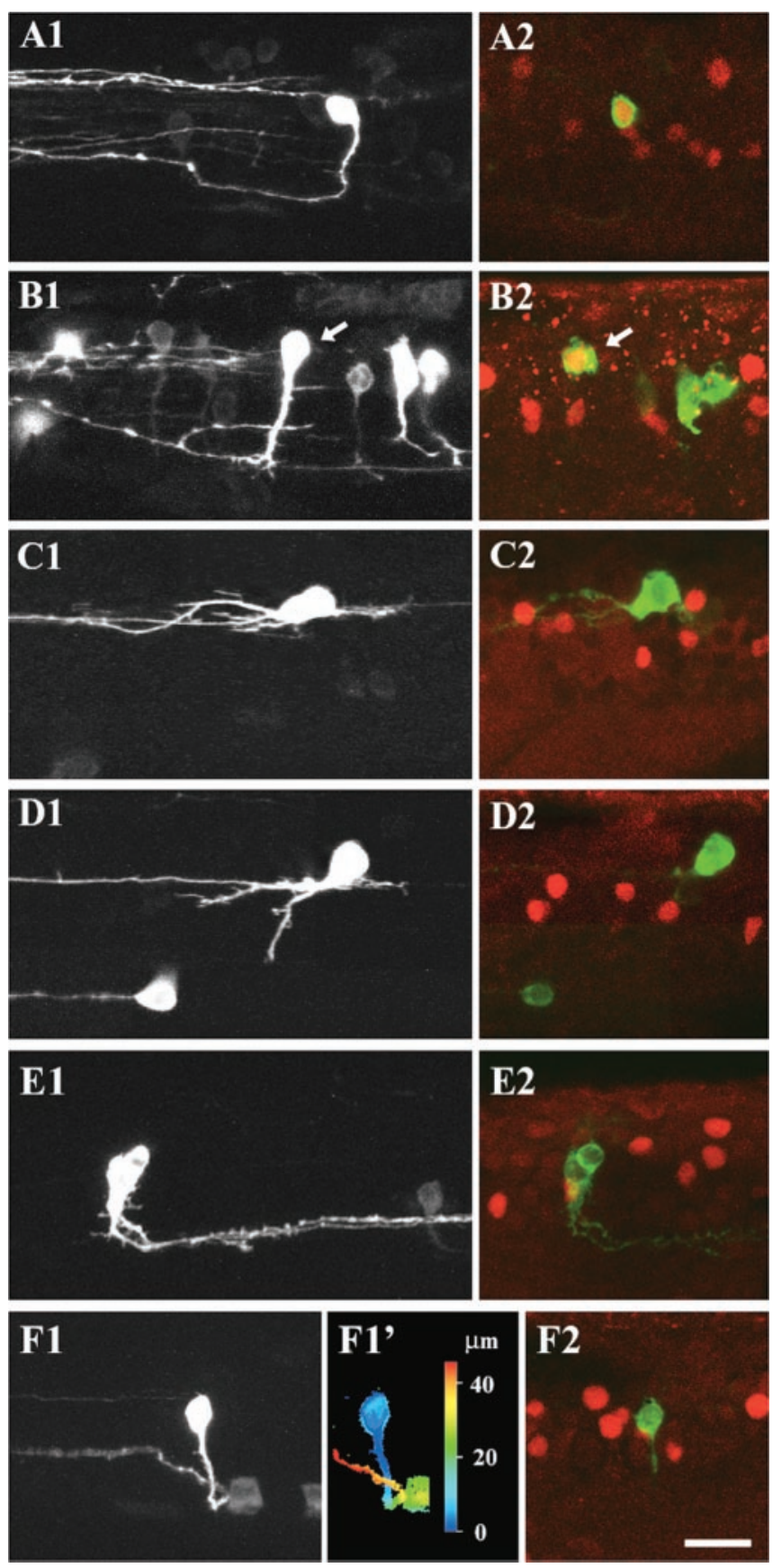

Figure 4. Only CiA interneurons are positive for En1. A variety of types of interneurons were stochastically labeled by cameleon (CAM; a derivative of GFP) using pan-neuronal promoter constructs. Images were taken from 32-36 hr embryos. A1-F1 (left) are images of living CAMlabeled neurons (from stacks of optical sections), whereas A2-F2 (right) are corresponding images of the anti-En1 counterstaining (in a stack of $1-3$ optical sections), with the CAM signal in green and the En 1 signal in red. $A 1, A 2, A$ CiA interneuron is labeled by CAM (A1), and the cell is positive for $E n 1(A 2) . B 1, B 2$, Another CiA interneuron labeled by $C A M$ ( $B 1$, arrow) and positive for $E n 1$ ( $B 2$, arrow). Other types of cells are also labeled by CAM in $B 1$ (cells located in the right part of the figure), and these cells are negative for En1. C1, C2, An ascending DoLA interneuron is labeled by CAM (C1), and the cell is negative for $\operatorname{En} 1(C 2)$. D1, D2, Both a DoLA interneuron (dorsally) and a KA interneuron are labeled by CAM, and both are negative for En1. E1, E2, Two adjacent ipsilateral descending interneurons are labeled by CAM, and both are negative for En1 (note the lack of red staining of the nuclei, which stand out in the center of the labeled somata because of exclusion of the green (AM expression) $F 1_{1} F 1^{\prime}, F 2$, A commissural interneuron labeled by CAM (F1) is negative for En1 (F2). $F 1^{\prime}$ is a depth coded image to show that the commissural axon is contralateral ( $30-40 \mu \mathrm{m}$ distant) to the soma. Scale bar, $20 \mu \mathrm{m}$. 

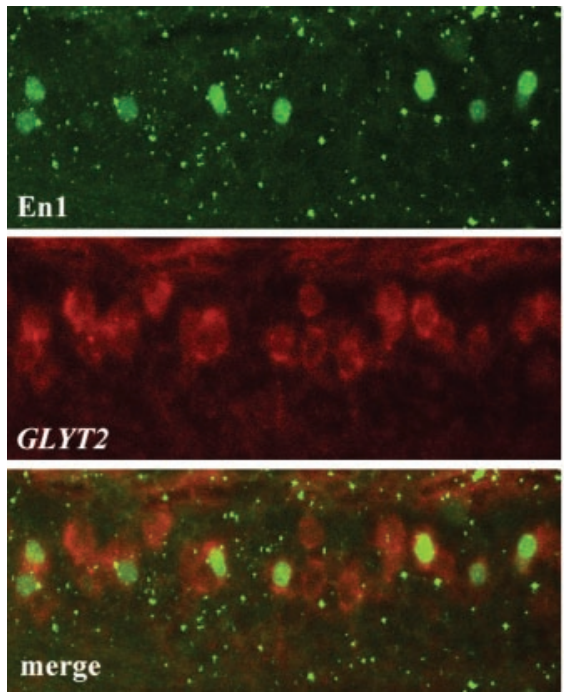
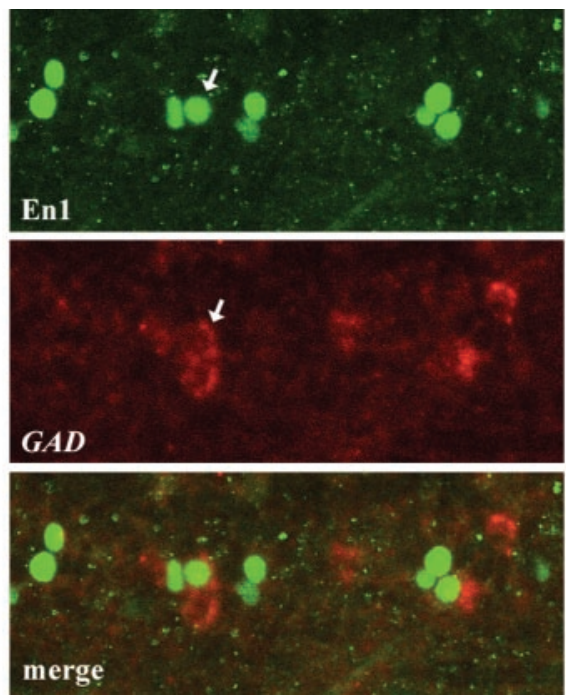
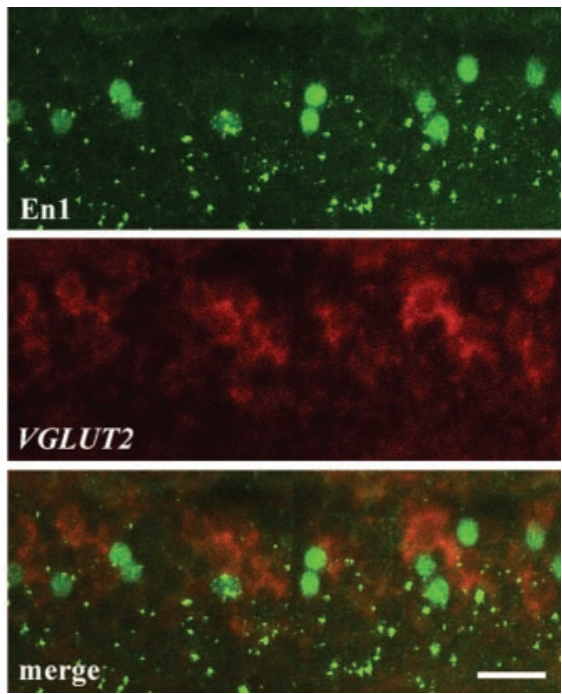

Figure 5. En1-positive interneurons are likely to be glycinergic. Images were taken from $32 \mathrm{hr}$ embryos. The left panels show dual staining of En 1 and GLYT2. All of the En1-positive interneurons are also positive for GLYT2. The center panels show dual staining of En1 and GAD (a mixture of GAD65 and GAD67). Some of En1-positive interneurons are positive for GAD (arrows). The right panels show dual staining of En1 and VGLUT2 (a mixture of VGLUT2.1 and VGLUT2.2). None of the En1-positive interneurons are positive for VGLUT2. Scale bar, $20 \mu m$.

fined by their far ventral and medial somata, which made contact with the central canal (Fig. 4D1, ventral cell). CiA interneurons had the major features described previously, although their dendrites and caudal axonal branches were not often prominent at this stage (Fig. 4A1,B1). Eighteen morphologically identified CiA interneurons were examined in total, and all 18 were positive for En1 (Fig. 4A2,B2). Eight morphologically identified DoLA interneurons were examined, and all were negative for En1 (Fig. $4 C, D)$. En 1 is not expressed in the region where KA interneurons are located, indicating that they are not positive for En1. We confirmed this in two KA interneurons that we found negative for En1 (Fig. 4D2).

Neurons other than ipsilateral ascending interneurons were less systematically investigated. However, many interneuron types were labeled in the experiments described above, and we never found En1-positive cells among non-CiA-type interneurons. These negatives included ipsilateral descending interneurons $(n=6)$ (Fig. $4 E)$, commissural interneurons $(n=12)$ (Fig. $4 F)$, motoneurons $(n=4)$, and $\mathrm{RB}$ sensory neurons $(n=7)$.

Taken together, the results indicate that all of the morphologically identified CiA interneurons are positive for En1 and that CiA interneurons are the only En1-positive interneurons, at least by $36 \mathrm{hr}$. The previous results from 3-d-old fish using the En 1GFP BAC expression suggest that the same conclusion also applies later in development.

\section{En1-positive interneurons are likely to be glycinergic}

We examined the neurotransmitter phenotype of the En1positive neurons by dual staining with En 1 and with genes with expression patterns that are tightly related to a particular neurotransmitter phenotype. The transmitter markers included GLYT2 for glycinergic neurons, the VGLUT genes for glutamatergic neurons, and GAD 65/67 for GABAergic neurons (Martin et al., 1998). The zebrafish GLYT2 and three types of VGLUT (VGLUT1, VGLUT2.1, and VGLUT2.2) were cloned by us, and the details will be described elsewhere.

The dual staining with GLYT2 indicated that nearly all of the En1-positive interneurons were positive for GLYT2. An example of a $32 \mathrm{hr}$ embryo is shown in Figure 5 (left panels), which shows that all of the En1-positive nuclei (green) are surrounded by
GLYT2-positive cytoplasm (red). In a few cases, we observed En1-positive interneurons that were not accompanied by a detectable level of GLYT2 staining (data not shown). The En1 staining in these cells was always very weak, and thus we speculate that these cells were early in their differentiation and would express GLYT2 later. Consistent with this, weakly labeled En1-positive cells at even earlier stages (20-22 hr) were often not accompanied by a detectable level of GLYT2 signal. The En1-positive interneurons were also positive for GLYT2 at 2, 3, and $4 \mathrm{~d}$ (data not shown).

Dual staining with GAD (a mixture of GAD65 and GAD67) at $32 \mathrm{hr}$ showed that a small subset ( $20 \%)$ of En1-positive interneurons appeared positive for $G A D$ (Fig. 5, center panels). Most of these showed faint $G A D$ staining, which was sometimes hard to distinguish from background; rarely, a strongly $G A D$-positive En1 neuron was observed (Fig. 5, arrows). At this early stage, the majority of $G A D$-positive cells in intermediate regions of cord are also positive for GLYT2 (S. Higashijima, G. Mandel, and J. R. Fetcho, unpublished observations), and, indeed, triple staining of En1, GLYT2, and GAD showed that these GAD-positive En1positive interneurons were also positive for GLYT2 (data not shown). Dual staining of En1 and VGLUT2 (a mixture of VGLUT2.1 and VGLUT2.2; VGLUT1 is not expressed in embryonic spinal cord) at $32 \mathrm{hr}$ showed that none of the En1-positive interneurons were positive for VLGUT2 (Fig. 5, right panels).

These results show that En1-positive interneurons are positive for GLYT2, strongly indicating that En1-positive cells (CiA interneurons) are glycinergic inhibitory interneurons. They may also simultaneously use GABA as a neurotransmitter at early stages. None were positive for the glutamatergic markers.

\section{Electrophysiology of the En1-positive CiA neurons}

The morphology and transmitter phenotype of the En1-positive CiA neurons suggested that they might correspond to a neuron involved in gating the flow of information through sensory reflex pathways that was predicted in studies of Xenopus many years ago (Sillar and Roberts, 1988, 1992a,b). Our anatomical data showed that the CiA interneurons appeared to contact CoPA interneurons. Several lines of evidence indicate that the CoPA interneurons are sensory interneurons that relay excitation from RB sen- 
A. $\mathrm{En} 1(\mathrm{CiA})$ during swimming
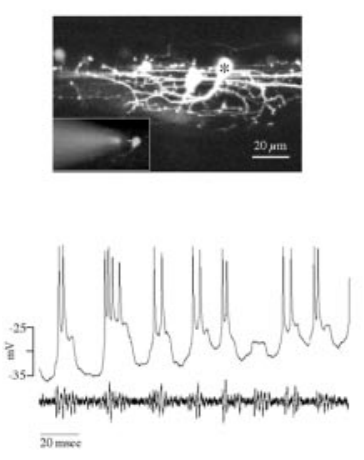

B. CoPA during swimming
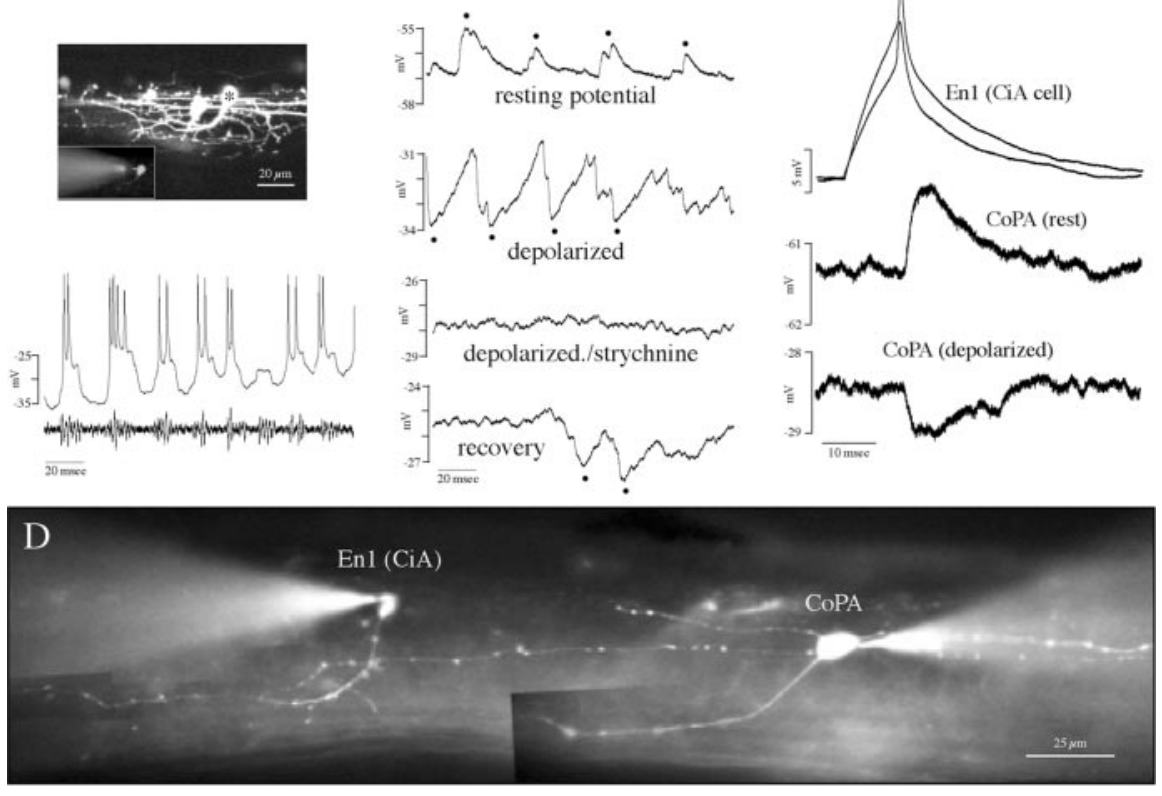

Figure 6. Activity of the En1-positive interneurons and their connections with CoPA interneurons. A, Patch recording from a CiA interneuron labeled by the En1-GFP BAC construct. The top panel shows an image of the GFP-labeled, patched cell, which is marked by an asterisk. The inset shows the same neuron filled with rhodamine from the patch electrode, which enters from the left side of the figure. The bottom panel shows the activity recorded from the CiA neuron (top) and a peripheral motor nerve (bottom) on the same side of the body as the cell. Fictive swimming was induced by a light stimulus and is evident in the rhythmic bursts recorded from the motor nerve. The CiA interneuron initially depolarized to approximately $-35 \mathrm{mV}$ from its resting potential of -54 (data not shown) and then showed the rhythmic depolarizations evident in the figure, which were usually accompanied by firing of action potentials. These depolarizations were in phase with the bursts of motor activity in the ipsilateral motor nerve. $B$, Recordings from a CoPA during episodes of swimming. All panels are from the same interneuron. Top panel, During swimming, a CoPA interneuron was depolarized to $-57 \mathrm{mV}$ from its resting potential of $-61 \mathrm{mV}$. Rhythmic, fast depolarizing potentials at the frequency of swimming (some of which are marked by dots) are superimposed on the sustained depolarization. Second panel, When the COPA is held depolarized (in this case to $\sim-28 \mathrm{mV}$ ), the fast potentials (dots) during swimming invert. Third panel, The inverted fast potentials in a depolarized CoPA during swimming are absent in strychnine, indicating they are glycinergic. Fourth panel, Recovery of the inverted fast potentials (dots) after washout of strychnine. C, Simultaneous patch recording from a CiA interneuron and a CoPA interneuron. Top, Action potentials elicited by current injection into a CiA neuron in two different trials. The responses of the CoPA neuron in the two cases are shown below. When the CoPA was at rest (middle), firing the CiA interneuron produced a short latency, depolarizing PSP, similar in time course to those in a CoPA during swimming. When the CoPA was depolarized from rest to $-28.5 \mathrm{mV}$ (bottom), firing the CiA led to an inverted PSP in the COPA. D, Photomontage of a pair of cells, the physiology of which is shown in C, taken immediately after the recordings. The CiA is on the left, and the CoPA is on the right. In this case, the CoPA was caudal to the $\mathrm{CiA}$, but the descending branch of the CiA axon had processes that approached the COPA soma and dendrite, consistent with the monosynaptic connection indicated by the pairwise physiology. The patch electrodes enter from the left and right sides of the figure.

sory neurons innervating the skin to the opposite side of the body (Gleason et al., 2003). In three experiments, we confirmed this connection between RB neurons and the CoPA interneurons by pairwise patch recording from an $\mathrm{RB}$ and a CoPA interneuron. In each case, firing the RB neurons produced a short latency, EPSP in the CoPA interneuron (data not shown), consistent with the connection suggested by prior morphological and physiological evidence. The major features of the CoPA neurons are similar to the dorsolateral commissural interneurons in Xenopus, which are known to receive a rhythmic inhibition during swimming that gates the flow of sensory information through the cutaneous reflex pathway (Sillar and Roberts, 1992a,b). The observation that the $\mathrm{CiA}$ interneurons were inhibitory and contacted the CoPA neurons suggested a possible role in inhibiting flow through the CoPA sensory pathway. We examined the electrophysiology of the $\mathrm{CiA}$ and CoPA interneurons to explore whether the CiA cells had the predicted features of a neuron involved in gating activity in the CoPA pathway during swimming.
We first examined whether the CiA neurons were active during fictive swimming. In 4- to 5-d-old fish, we targeted for patch recording $\mathrm{CiA}$ neurons labeled by GFP expression with the En1-GFP construct and simultaneously recorded from peripheral nerves or from a motoneuron to monitor the motor pattern. All 20 of the $\mathrm{CiA}$ interneurons we examined depolarized during a swimming episode and had rhythmic oscillations of membrane potential superimposed on a sustained depolarization (Fig. 6A). For 14 of the interneurons, we simultaneously monitored the motor pattern by recording from an ipsilateral motor neuron $(n=7)$ or peripheral nerve $(n=7)$. The rhythmic depolarizations of the membrane potential in the CiA interneurons during swimming occurred mostly in phase with ipsilateral motor activity recorded from peripheral nerves or motoneurons. Seventeen of the $20 \mathrm{CiAs}$ fired during at least some of the swimming episodes, often firing multiple spikes in each cycle of activity, like the neuron in Figure $6 A$.

Patch recordings from 15 CoPA interneurons during swimming showed that they also were tonically depolarized during a swimming episode. Unlike the CiA neurons, however, none of the CoPA interneurons fired action potentials during swimming. A series of faster depolarizing PSPs were superimposed on the tonic depolarization of the CoPAs throughout swimming (Fig. 6B). These faster depolarizations showed some rhythmicity during swimming, but not all of them were always tightly coupled with the swimming rhythm. The depolarizations reversed before reaching spike threshold in 9 of 10 interneurons where the synaptic reversal potential was tested (as in Fig. 6B), suggesting that they were likely chloridedependent PSPs. These PSPs were reversibly blocked by application of strychnine in four of five neurons tested (Fig. 6B), supporting the conclusion that they were glycinergic, chloride-dependent PSPs.

The previous results suggested that the CoPA interneurons receive glycinergic PSPs during swimming that could serve to block their activation by sensory pathways during a swimming episode. The only known candidates for interneurons in zebrafish spinal cord that might provide this inhibition are the commissural glycinergic interneurons or the ipsilaterally projecting $\mathrm{CiA}$ (En1) interneurons. The CiA axons are more dorsal than the axons of commissural neurons and are apposed to the dendrites and somata of the CoPA neurons, making them the most likely candidates for providing the inhibition.

This potential connection between the CiA (En1) and CoPA interneuron was examined by pairwise recording from the two cell types in three experiments. Figure $6 C$ shows a representative example. In each case, firing the CiA neurons produced short latency depolarizing PSPs in the CoPA interneurons, with aver- 
age synaptic delays of $0.46,0.63$, and $0.91 \mathrm{msec}(\mathrm{SD}=0.1,0.08$, and 0.07 , respectively; $n=9$ for each cell) in the three cells. The postsynaptic responses in the CoPAs followed firing of the CiA without latency jitter at frequencies of stimulation that fatigued the postsynaptic response. The very short latency and absence of latency jitter support the conclusion that the connection is monosynaptic (Fetcho,1990). In two of the three pairs, we tested the reversal potential of the PSPs (as in Fig. 6C) and found that they reversed at negative membrane potentials, similar to potentials that reversed the strychnine-sensitive PSPs in CoPAs during swimming. In both of these pairs, we also applied strychnine, which blocked the synaptic connection. The negative reversal potential and strychnine sensitivity indicate that these are IPSPs.

If we had effectively dialyzed the cell with the patch solution, which contained low chloride, we would expect chloridedependent IPSPs to be hyperpolarizing because of a calculated reversal potential negative to rest $(-90 \mathrm{mV})$. The depolarizing responses we observed suggest that the normal chloride reversal potential is higher than rest and that the synapses were on the long dendrites, at a location that was not effectively dialyzed. Images of the recorded neurons, which were filled with dye from the patch electrodes, showed that the CiA neurons had either ascending or descending branches of their axons apposed to the dendrites of postsynaptic CoPA cells, providing additional support for the monosynaptic connection indicated by the physiology.

In summary, both the electrophysiological and morphological data support the conclusion that the CiA (En1) interneurons monosynaptically inhibit the CoPA neurons via glycinergic synapses. The activity of the CiA cells during swimming likely accounts for the strychnine-sensitive PSPs we observed in the CoPA interneurons during swimming. Individual CiA neurons, however, are rhythmically active in swimming, whereas the CoPA neurons received strychnine-sensitive PSPs that are not as tightly coupled with the swimming rhythm. This is likely explained by the convergence of the long ascending (and descending processes) of many CiA neurons from all along the body onto individual CoPA interneurons. The connectivity and activity pattern of the $\mathrm{CiA}$ neurons is consistent with a role in blocking the flow of activity through the CoPA sensory pathway during rhythmic swimming movements.

The branching pattern of the CiA interneurons suggested possible contacts with more ventral neurons, including motoneurons and other interneurons. We tested many (over 30) pairs of $\mathrm{CiA}$ and ventral cells for connections by stimulating a CiA while recording with whole-cell recording from neurons with somata that were adjacent to $\mathrm{CiA}$ processes. We found three cells that received short latency, IPSPs after firing of a CiA. One of these, shown in Figure 7, was a motoneuron that showed a synaptic response after extracellular stimulation of a CiA via a loose cellattached patch. The other two were descending interneurons that responded to CiAs activated via current injection during pairwise whole-cell recording. The latencies of these connections were short (mean: 0.76 msec for the motoneuron and 0.68 and 0.75 for the CiDs; SD: $0.11,0.16$, and 0.13 , respectively; $n=10$ for each cell), consistent with a monosynaptic input from the CiA. Unlike the CoPA interneurons, the PSPs were hyperpolarizing from rest. This is likely a consequence of the somatic location of synapses on the motoneurons and descending interneurons, in a region where the cell was effectively dialyzed by the patch pipette and synaptic reversal potential was determined by the low-chloride concentration in our intracellular solution. CoPA connections were often on dendrites further from the somatic recording site.
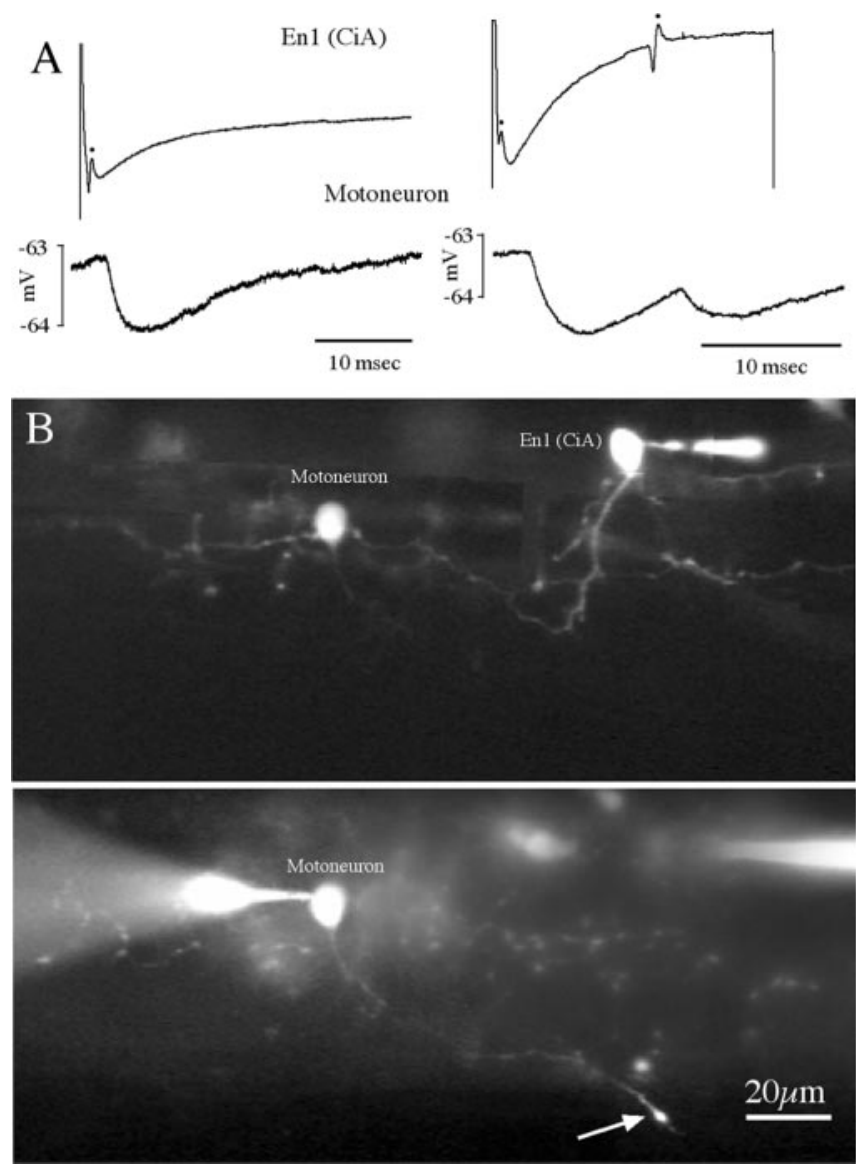

Figure 7. $\quad \mathrm{En} 1(\mathrm{CiA})$ interneurons inhibit motoneurons. A, Pairwise recordings of an En1 interneuron and a postsynaptic motoneuron, with En1 recordings on the top and motoneuron responses on the bottom. Left, Current application to a loose patch on the En1 neuron fires the cell, as evident in the extracellular recording of the spike-generated current : The spike is followed at short latency by an IPSP in the motoneuron. Right, Two successive spikes in the En1 neuron (dots) are each associated with short latency IPSPs in the motoneuron. B, The morphology of the two cells, the physiology of which is shown in $A$. The top panel shows a montage of a GFP image showing the En 1 cell and its process that runs adjacent to the motoneuron at the left, the bright soma of which is visible because of cross excitation and bleedthrough of the rhodamine in the cell. The bottom panel shows a montage of the same region with a rhodamine filter set to demonstrate that the axon of the motoneuron exits in the ventral root (arrow). Patch pipettes are visible entering from the right in the top image and from the left and right in the bottom one. Both are lateral views of spinal cord with rostral to the left.

The difficulty in finding connections with ventral neurons was likely a combination of a small number of postsynaptic cells in the motor column contacted by each CiA, along with possible disruption of somatic connections by the positive pressure applied in the process of approaching the postsynaptic neuron. Connections with CoPA interneurons were found more easily, possibly because of the greater convergence of engrailed neurons onto the CoPA cells along with the dendritic locations of many of their contacts.

\section{Discussion}

We set out to define the morphology and functional properties of the spinal neurons that express En1 in zebrafish, with the hope that studies of a relatively simple model system might reveal a primitive link between transcription factor and interneuron type (Saueressig et al., 1999; Briscoe et al., 2000; Jessell, 2000; MoranRivard et al., 2001; Pierani et al., 2001; Sharma and Peng, 2001; Goulding et al., 2002). We found such a link. All of the En1- 
positive neurons in zebrafish are ipsilateral ascending glycinergic interneurons with a distinctive morphology. None of the other known classes of interneuron are En1 positive.

The En 1 cells in zebrafish are similar in all major morphological respects to the ascending interneurons in Xenopus tadpoles, which are also marked by En1 (Li et al., 2004). The soma location, dendritic arbors, and long ascending axon with a descending branch are strikingly similar in both species. The En1 neurons in the two species are also glycinergic.

Several features of the morphology and transmitter phenotype of the zebrafish neurons are also shared by the interneurons marked by En 1 expression in mice (Saueressig et al., 1999). The cell bodies of both are located dorsal to the motor column. The axons arise from the ventral aspect of their somata and extend ventrally and then rostrally, running in the ventrolateral fasiculus in mice and in a comparable location just lateral to the motor column in zebrafish. In both species, the axons appear to extend dorsally as they ascend through the region of the motor column, leading to a picture in lateral view that looks very similar in zebrafish and mice (compare our Fig. $2 C 1$ with Fig. $2 \mathrm{D}$ in the study by Saueressig et al., 1999). The axons of the population of En1positive cells in mice form a crescent lateral to the motor column, with some axons dorsal to it. The trajectory of the initial portion of the axons in zebrafish also spreads them along the lateral surface of the motor column. A major difference, however, is that the axons of the En 1 cells in mice are short because they are backfilled only a couple of segments from an injection site. In zebrafish, they extend a long distance, running to the brain in an axon tract dorsal to the motor column, in a position similar to the most dorsal axons in mice. The En1 neurons in mice and chicks are positive for GABA markers early in life, but there is reason to think that they later switch to a glycinergic phenotype (Antal et al., 1994; Berki et al., 1995; Saueressig et al., 1999; Wenner and O'Donovan, 1999; Wenner et al., 2000; Nakayama et al., 2002). A similar switch is also suggested by the early dual staining for GABA and glycinergic markers of the En1 neurons in zebrafish. En1 expression thus marks interneurons with an overall morphology and transmitter phenotype that has been conserved in widely divergent groups of vertebrates, including fish, frog tadpoles, and mice.

The inhibition from En1-positive interneurons plays several functional roles in tadpoles and fish. Evidence from Xenopus tadpoles over 10 years ago showed that the throughput in sensory reflex pathways is gated by ipsilateral inhibition during swimming, so that when an animal is bending to one side during swimming, activation of motor output on the opposite side by sensory pathways is blocked by inhibition of commissural excitatory sensory interneurons (Sillar and Roberts, 1988, 1992a,b). Similar gating of sensory pathways is present in many motor systems, but the cells mediating it had not been identified in any vertebrate, until their recent discovery in Xenopus tadpoles (Li et al., 2002). We independently found them in zebrafish in the course of our studies of the En1-positive interneurons. The En1positive, CiA, neurons in zebrafish are rhythmically driven during swimming and monosynaptically inhibit CoPA interneurons. The CoPA neurons are commissural interneurons that are excited by RB sensory neurons and stain for vesicular glutamate transporters (Higashijima, M. Schaefer, M. Masino, and Fetcho, unpublished observations), indicating that they relay sensory excitation from one side of the body to the other. The inhibition of the CoPA interneurons by the CiA cells during swimming episodes could block the flow of excitation through this sensory pathway during swimming. This gating of the CoPA sensory pathway during swimming is analogous to the more extensively studied gating in the pathway for reflex bending in Xenopus ( Li et al., 2002).

A role in sensory gating is not the only function of the En1 neurons. Individual CiA interneurons in zebrafish have axonal arbors that branch widely in the spinal cord, both in dorsal, sensory regions as well as ventral, motor ones. Their axons are directly apposed to the somata of motoneurons, and their widely distributed branches raise the possibility that they contact many different cell types spread throughout much of the dorsoventral extent of spinal cord. This is supported by our data showing that they directly inhibit both motoneurons and descending interneurons. These observations are consistent with recent, more extensive physiological studies of the inhibitory ascending interneurons that perform sensory gating in Xenopus, which indicate that these interneurons are responsible for inhibition of many ipsilateral cell types, including interneurons in the central pattern generator for swimming, as well as the motoneurons themselves ( $\mathrm{Li}$ et al., 2004). These Xenopus neurons are En1 positive, like their ascending counterpart in zebrafish.

Taken together, the evidence from zebrafish and Xenopus indicates that En 1 marks a single class of multifunctional, ipsilateral, glycinergic interneurons in both species. The En1 interneurons are the only known glycinergic neurons with projections in ipsilateral spinal cord of zebrafish larvae (Higashijima, Schaefer, and Fetcho, unpublished observations). The only other known glycinergic cell types in the spinal cord are the En1-negative commissural interneurons. We cannot totally rule out the possibility that other ipsilateral inhibitory cell types exist but have been missed in our studies of the transmitter phenotypes of the known classes of interneurons. Based on the available evidence, however, the En 1 neurons appear to provide all of the ipsilateral glycinergic inhibition in spinal circuits of zebrafish at the stages we studied them.

The common ancestor of fish and frogs is ancient, suggesting that the shared features of the engrailed interneurons are primitive ones for vertebrates. We propose that En 1 expression marked the only glycinergic cell type with ipsilateral projections in the spinal cord of early vertebrates. These interneurons were multifunctional inhibitory neurons that played roles in producing and shaping rhythmic motor output, as well as in gating the flow of sensory information during locomotion. This primitive vertebrate arrangement is retained in the larvae of aquatic vertebrates such as zebrafish and Xenopus, in which the neurons provide all of the ipsilateral glycinergic inhibition early in life.

We know less about the organization of the spinal cords of fish and frogs later in life. The animals grow considerably through life, and the organization of the interneurons might change as they grow. Larval zebrafish have relatively few interneuron types in the spinal cord, but the cells are sufficient for the animals to produce the range of coordinated movements needed for the free swimming larvae to survive. Although some of the classes of interneurons evident in larvae are also present in adult fishes (Ritter et al., 2001), it is, nonetheless, possible that the En1 interneurons in larvae differentiate into more specialized subtypes as the animals grow to adulthood. This might, for example, be directed by transcription factors that differentially affect En 1 cells located at somewhat different dorsoventral positions in the spinal cord.

Mammals and birds have a more diverse array of ipsilateral inhibitory neurons than larval fishes and amphibians. The primitive multifunctional En1 interneurons evident in zebrafish and Xenopus may have specialized during evolution to give rise to the several different glycinergic cell types with ipsilateral connections 
in mammalian and avian spinal cords. Morphological and physiological evidence from chicks and mice indicates that En 1 marks more than one class of interneuron, including the well studied Renshaw cells, which provide feedback inhibition of motoneurons (Wenner et al., 2000; Sapir et al., 2004). The En1 cells in zebrafish and Xenopus also inhibit motoneurons, making them a candidate for a cell type ancestral to Renshaw neurons. Indeed, the morphological and functional similarities between the En1positive cells in zebrafish and those in mice and chicks make the CiA neurons in fish the best (and at present only) candidate for a cell type homologous to the En1 neurons in more complex vertebrates.

If the En 1 neurons primitively provided all of the ipsilateral inhibition, as appears to be the case based on fish and frogs, we might expect that all of the ipsilateral glycinergic premotor interneurons in birds and mammals will be En1 positive early in their development. This would include not only the Renshaw cells, but also the still unidentified interneurons involved in sensory gating in birds and mammals, the Ia inhibitory interneurons in reflex pathways, the group I nonreciprocal inhibitory interneurons (Ib), as well as the inhibitory interneurons in the mammalian central pattern generator that produce flexor and extensor alternation on one side of the body (Baldissera et al., 1981; Grillner, 1981; Burke and Fleshman, 1986; Kiehn and Kjaerulff, 1998; Jankowska and Hammar, 2002). Not all of the En 1 cell types have been identified in mammals, but recent work suggests that the En1 population includes the Ia and Ib inhibitory interneurons along with the Renshaw cells (Alvarez et al., 2003; Sapir et al., 2004). This is consistent with the possibility that a multifunctional class of neurons like the En1 cells in zebrafish and frog tadpoles may have been the precursors of several more specialized cell types in mammals.

In summary, the evidence that En 1 marks a unique functional class of neurons in larval fishes and frogs is consistent with the idea that transcription factors define a primitive "ground state" of spinal interneurons (Goulding et al., 2002). The spinal cord in larvae of swimming vertebrates is simple, with a small number of distinct functional classes of neurons. Such a simple organization could be set up via expression of a small number of transcription factors. The early development of spinal cord may serve to define this primitive functional organization of interneurons, which forms a developmental and evolutionary foundation on which more complex systems are built.

\section{References}

Alvarez FJ, Jonas P, Sapir T, Gieman EJ, Hartley R, Todd AJ, Goulding M (2003) Postnatal phenotype and localization of spinal cord V1 derived interneurons. Soc Neurosci Abstr 29:39.3.

Antal M, Berki AC, Horvath L, O’Donovan MJ (1994) Developmental changes in the distribution of gamma-aminobutyric acidimmunoreactive neurons in the embryonic chick lumbosacral spinal cord. J Comp Neurol 343:228-236.

Baldissera F, Hultborn H, Illert M (1981) Integration in spinal neuronal systems. In: Handbook of physiology, Sec 1, The nervous system, Vol II, Motor control (Brooks VB, ed), pp 509-595. Bethesda, MD: American Physiological Society.

Berki AC, O’Donovan MJ, Antal M (1995) Developmental expression of glycine immunoreactivity and its colocalization with GABA in the embryonic chick lumbosacral spinal cord. J Comp Neurol 362:583-596.

Bernhardt RR, Chitnis AB, Lindamer L, Kuwada JY (1990) Identification of spinal neurons in the embryonic and larval zebrafish. J Comp Neurol 302:603-616.

Briscoe J, Pierani A, Jessell TM, Ericson J (2000) A homeodomain protein code specifies progenitor cell identity and neuronal fate in the ventral neural tube. Cell 101:435-445.

Burke RE, Fleshman JW (1986) Strategies to identify interneurons involved in locomotor pattern generation in the mammalian spinal cord. In: Neurobiology of vertebrate locomotion (Grillner S, Stein PSG, Forssberg H, Herman RM, eds), pp 245-267. London: Macmillian.

Burrill JD, Moran L, Goulding MD, Saueressig H (1997) PAX2 is expressed in multiple spinal cord interneurons, including a population of EN1+ interneurons that require PAX6 for their development. Development 124:4493-4503.

Davis CA, Holmyard DP, Millen KJ, Joyner AL (1991) Examining pattern formation in mouse, chicken and frog embryos with an En-specific antiserum. Development 111:287-298.

Drapeau P, Ali DW, Buss RR, Saint-Amant L (1999) In vivo recording from identifiable neurons of the locomotor network in the developing zebrafish. J Neurosci Methods 88:1-13.

Ekker M, Wegner J, Akimenko MA, Westerfield M (1992) Coordinate embryonic expression of three zebrafish engrailed genes. Development 116:1001-1010.

Fetcho JR (1990) Morphological variability, segmental relationships, and functional role of a class of commissural interneurons in the spinal cord of goldfish. J Comp Neurol 299:283-298.

Fetcho JR (2001) Optical and genetic approaches toward understanding spinal circuits. In: Motor neurobiology of the spinal cord (Cope TC, ed), pp 3-20. Boca Raton, FL.: CRC.

Fetcho JR, O'Malley DM (1995) Visualization of active neural circuitry in the spinal-cord of intact zebrafish. J Neurophysiol 73:399-406.

Fjose A, Njolstad PR, Nornes S, Molven A, Krauss S (1992) Structure and early embryonic expression of the zebrafish engrailed-2 gene. Mech Dev 39:51-62.

Force A, Lynch M, Pickett FB, Amores A, Yan YL, Postlethwait J (1999) Preservation of duplicate genes by complementary, degenerative mutations. Genetics 151:1531-1545.

Gleason MR, Higashijima S, Dallman J, Liu K, Mandel G, Fetcho JR (2003) Translocation of CaM kinase II to synaptic sites in vivo. Nat Neurosci 6:217-218.

Goulding M, Lanuza G, Sapir T, Narayan S (2002) The formation of sensorimotor circuits. Curr Opin Neurobiol 12:508-515.

Grillner S (1981) Control of locomotion in bipeds, tetrapods, and fish. In: Handbook of physiology, Sect I, The nervous system II (Brooks VB, ed), pp 1179-1236. Washington, DC: American Physiological Society.

Hale ME, Ritter DA, Fetcho JR (2001) A confocal study of spinal interneurons in living larval zebrafish. J Comp Neurol 437:1-16.

Halloran MC, Sato-Maeda M, Warren JT, Su F, Lele Z, Krone PH, Kuwada JY, Shoji W (2000) Laser-induced gene expression in specific cells of transgenic zebrafish. Development 127:1953-1960.

Hatta K, Bremiller R, Westerfield M, Kimmel CB (1991) Diversity of expression of engrailed-like antigens in zebrafish. Development 112:821-832.

Hieber V, Dai X, Foreman M, Goldman D (1998) Induction of alpha1tubulin gene expression during development and regeneration of the fish central nervous system. J Neurobiol 37:429-440.

Higashijima S, Okamoto H, Ueno N, Hotta Y, Eguchi G (1997) Highfrequency generation of transgenic zebrafish which reliable express GFP in whole muscles or the whole body by using promoters of zebrafish origin. Dev Biol 192:289-299.

Higashijima SI, Masino MA, Mandel G, Fetcho JR (2003) Imaging neuronal activity during zebrafish behavior with a genetically encoded calcium indicator. J Neurophysiol 90:3986-3997.

Holland PW, Williams NA (1990) Conservation of engrailed-like homeobox sequences during vertebrate evolution. FEBS Lett 277:250-252.

Jankowska E, Hammar I (2002) Spinal interneurones; how can studies in animals contribute to the understanding of spinal interneuronal systems in man? Brain Res Rev 40:19-28.

Jessell TM (2000) Neuronal specification in the spinal cord: inductive signals and transcriptional codes. Nat Rev Genet 1:20-29.

Jessen JR, Meng A, McFarlane RJ, Paw BH, Zon LI, Smith GR, Lin S (1998) Modification of bacterial artificial chromosomes through chi-stimulated homologous recombination and its application in zebrafish transgenesis. Proc Natl Acad Sci USA 95:5121-5126.

Joyner AL, Martin GR (1987) En-1 and En-2, two mouse genes with sequence homology to the Drosophila engrailed gene: expression during embryogenesis. Genes Dev 1:29-38.

Kiehn O, Kjaerulff O (1998) Distribution of central pattern generators for rhythmic motor outputs in the spinal cord of limbed vertebrates. Ann NY Acad Sci 860:110-129. 
Kiehn O, Kullander K (2004) Central pattern generators deciphered by molecular genetics. Neuron 41:317-321.

Kuwada JY, Bernhardt RR, Nguyen N (1990) Development of spinal neurons and tracts in the zebrafish embryo. J Comp Neurol 302:617-628.

Legendre P, Korn H (1994) Glycinergic inhibitory synaptic currents and related receptor channels in the zebrafish brain. Eur J Neurosci 6:1544-1557.

Li WC, Soffe SR, Roberts A (2002) Spinal inhibitory neurons that modulate cutaneous sensory pathways during locomotion in a simple vertebrate. J Neurosci 22:10924-10934.

Li W-C, Higashijima S, Parry DM, Roberts A, Soffe SR (2004) Primitive roles for inhibitory interneurons in developing frog spinal cord. J Neurosci 24:5840-5848.

Martin SC, Heinrich G, Sandell JH (1998) Sequence and expression of glutamic acid decarboxylase isoforms in the developing zebrafish. J Comp Neurol 396:253-266.

Miyawaki A, Llopis J, Heim R, McCaffery JM, Adams JA, Ikura M, Tsien RY (1997) Fluorescent indicators for Ca2 + based on green fluorescent proteins and calmodulin. Nature 388:882-887.

Moran-Rivard L, Kagawa T, Saueressig H, Gross MK, Burrill J, Goulding M (2001) Evx1 is a postmitotic determinant of v0 interneuron identity in the spinal cord. Neuron 29:385-399.

Nakayama K, Nishimaru H, Kudo N (2002) Basis of changes in left-right coordination of rhythmic motor activity during development in the rat spinal cord. J Neurosci 22:10388-10398.

Park HC, Kim CH, Bae YK, Yeo SY, Kim SH, Hong SK, Shin J, Yoo KW, Hibi M, Hirano T, Miki N, Chitnis AB, Huh TL (2000) Analysis of upstream elements in the $\mathrm{HuC}$ promoter leads to the establishment of transgenic zebrafish with fluorescent neurons. Dev Biol 227:279-293.
Pierani A, Moran-Rivard L, Sunshine MJ, Littman DR, Goulding M, Jessell TM (2001) Control of interneuron fate in the developing spinal cord by the progenitor homeodomain protein Dbx1. Neuron 29:367-384.

Ritter DA, Bhatt DH, Fetcho JR (2001) In vivo imaging of zebrafish reveals differences in the spinal networks for escape and swimming movements. J Neurosci 21:8956-8965.

Sapir T, Geiman EJ, Wang Z, Velasquez T, Mitsui S, Yoshihara Y, Frank E, Alvarez FJ, Goulding M (2004) Pax6 and engrailed1 regulate two distinct aspects of renshaw cell development. J Neurosci 24:1255-1264.

Saueressig H, Burrill J, Goulding M (1999) Engrailed-1 and netrin-1 regulate axon pathfinding by association interneurons that project to motor neurons. Development 126:4201-4212.

Sharma K, Peng CY (2001) Spinal motor circuits: merging development and function. Neuron 29:321-324.

Sillar KT, Roberts A (1988) A neuronal mechanism for sensory gating during locomotion in a vertebrate. Nature 331:262-265.

Sillar KT, Roberts A (1992a) The role of premotor interneurons in phasedependent modulation of a cutaneous reflex during swimming in Xenopus laevis embryos. J Neurosci 12:1647-1657.

Sillar KT, Roberts A (1992b) Phase-dependent modulation of a cutaneous sensory pathway by glycinergic inhibition from the locomotor rhythm generator in Xenopus embryos. Eur J Neurosci 4:1022-1034.

Wenner P, O’Donovan MJ (1999) Identification of an interneuronal population that mediates recurrent inhibition of motoneurons in the developing chick spinal cord. J Neurosci 19:7557-7567.

Wenner P, O’Donovan MJ, Matise MP (2000) Topographical and physiological characterization of interneurons that express engrailed-1 in the embryonic chick spinal cord. J Neurophysiol 84:2651-2657. 